Produção, Caracterização e Aplicação de Carvão Ativado de Casca de Nozes para Adsorção de Azul de Metileno

\author{
de Costa, P. D.; Furmanski, L. M.; Dominguini, L.*
}

Rev. Virtual Quim., 2015, 7 (4), 1272-1285. Data de publicação na Web: 3 de março de 2015

http://www.uff.br/rvq

\title{
Production, Characterization and Application of Activated Carbon from Nutshell for Adsorption of Methylene Blue
}

\begin{abstract}
In this paper is reported the preparation of an activated carbon from an agricultural waste, rich in carbon, the nutshell. Physical activation by pyrolysis and physic-chemical activation with pyrolysis and chemical treatment were used for the preparation of the activated carbon. The material was characterized in relation to amount of carbon and ash, thermogravimetric analysis (TGA), Fourier transform infrared spectroscopy (FTIR), scanning electron microscopy (SEM) and surface area (BET). The results showed that the coal produced had carbon content above $78 \%$, with a surface area around $400 \mathrm{~m}^{2} \cdot \mathrm{g}^{-1}$ and high thermal stability and morphology honeycomb type. Tests for the adsorption capacity of methylene blue showed a capacity of $68 \mathrm{mg} \cdot \mathrm{g}^{-1}$ for the activated carbon produced without chemical treatment and a capacity of $104 \mathrm{mg} \cdot \mathrm{g}^{-1}$ for activated carbon treated with a chemical activation with $\mathrm{ZnCl}_{2}$. Thus, the material showed promise in the removal of methylene blue in aqueous solution.
\end{abstract}

Keywords: activated carbon; nutshell; adsorption; methylene blue.

\section{Resumo}

Neste trabalho, foi preparado um carvão ativado a partir de um resíduo agrícola, rico em carbono, a casca de noz. Ativação física por pirólise e ativação físico-química foram usadas para a preparação dos carvões ativados. O material foi caracterizado em relação ao teor de carbono e cinzas, TGA (análise termogravimétrica), FTIR (espectroscopia de infravermelho com transformada de Fourier), MEV (microscopia eletrônica de varredura) e BET (área superficial). Os resultados mostram que o carvão produzido apresenta um teor de carbono acima de $78 \%$, com uma área de superfície de cerca de $400 \mathrm{~m}^{2} \cdot \mathrm{g}^{-1} \mathrm{com}$ uma elevada estabilidade térmica e morfologia tipo favo de mel. Os testes de capacidade de adsorção de azul de metileno mostrou cerca de $68 \mathrm{mg} \cdot \mathrm{g}^{-1}$ para o carbono ativado produzido por meios físicos e $104 \mathrm{mg} \cdot \mathrm{g}^{-1}$ para ativação físico-química com $\mathrm{ZnCl}_{2}$. Assim, o material mostrou ser um promissor na remoção de azul de metileno em solução aquosa.

Palavras-chave: carvão ativado; casca de noz; adsorção; azul de metileno.

* Instituto Federal de Santa Catarina, Campus Criciúma, Rod. SC 443, km 1, CEP 88813-600, Vila Rica, Criciúma-SC.

Mlucas.dominguini@ifsc.edu.br

DOI: $\underline{10.5935 / 1984-6835.20150070}$ 


\section{Produção, Caracterização e Aplicação de Carvão Ativado de Casca de Nozes para Adsorção de Azul de Metileno \\ Patricia D. de Costa, ${ }^{a}$ Luana M. Furmanski, ${ }^{b}$ Lucas Dominguini ${ }^{\mathrm{c}, \mathrm{d}, *}$}

aUniversidade do Extremo Sul Catarinense, Programa de Pós-Graduação em Ciências Ambientais, CEP 88806-000, Criciúma-SC, Brasil.

bUniversidade do Extremo Sul Catarinense, Programa de Pós-Graduação em Ciência e Engenharia de Materiais, CEP 88806-000, Criciúma-SC, Brasil.

IInstituto Federal de Santa Catarina, Campus Criciúma, CEP 88813-600, Criciúma-SC, Brasil.

'Universidade Federal de Santa Catarina, Programa de Pós-Graduação em Engenharia Química, CEP 88040-970, Florianópolis-SC, Brasil.

* lucas.dominguini@ifsc.edu.br

\section{Introdução}

\section{Materiais e Método}

\subsection{Amostras}

2.1. Preparação do carvão ativado

2.3. Caracterização do carvão ativado

\section{Resultados e Discussões}

3.1. Caracterização física e química

3.2. Cinética de adsorção

3.3. Isoterma de adsorção

\section{Conclusões}

\section{Introdução}

A contaminação de um curso d'água nem sempre é facilmente detectável, uma vez que a maioria dos contaminantes não apresenta indícios visualmente identificáveis. A cor é o parâmetro que mais chama a atenção por meio do impacto visual proporcionado e que mais facilita a identificação da presença de uma contaminação.

Os efluentes têxteis quando despejados em curso d'água são exemplos de contaminações caracterizadas pela tonalidade de cor, visto que são coloridos devido a presença de corantes que não se fixaram nas fibras durante o processo de tingimento. ${ }^{1}$ Além disso, este tipo de 
indústria é normalmente caracterizada pelo elevado volume de água empregado em seu processo e, por consequência, pelas quantidades elevadas de efluentes gerados. ${ }^{2}$

O aumento na atenção em relação aos despejos dos efluentes industriais, tanto pelas indústrias e órgãos ambientais como pela sociedade em geral, elevou a quantidade de estudos direcionados a novos possíveis métodos de tratamento. Estes, normalmente aplicam os processos de precipitação, de troca iônica, de coagulação, osmose reversa e, principalmente, de adsorção. ${ }^{3}$

Em geral, a adsorção com materiais adsorventes alternativos, obtidos a partir de resíduos orgânicos, é um dos métodos considerados eficazes no processo de remoção de contaminantes em solução, sendo de baixo custo e de baixa complexidade. ${ }^{4-7} \mathrm{~A}$ adsorção é considerada de desempenho superior a outras técnicas devido à possibilidade de reutilização da água após o processo de purificação. Ainda, tratase de um processo de elevada flexibilidade, simplicidade, facilidade de operação e de insensibilidade aos compostos tóxicos. ${ }^{8}$ É uma das mais usadas porque as espécies de contaminantes são transferidos do efluente para uma fase sólida, reduzindo o volume de descarte ao mínimo. ${ }^{9,10}$

O carvão ativado é um dos adsorventes mais utilizados para a remoção de contaminantes de meios aquosos, devido a sua elevada capacidade de captura de moléculas por interação química e à alta taxa de remoção por causa de sua grande área superficial específica. ${ }^{11-13} \mathrm{~A}$ aplicação desse material no tratamento de efluentes está relacionada ainda à redução de material orgânico, principalmente de espécies que alteram a cor de efluentes. ${ }^{14,15}$

Grande parte dos estudos com adsorventes buscam o uso de materiais alternativos de baixo custo e que possam ser aplicados na produção de carvão ativado, em substituição ao carvão mineral. ${ }^{16}$ Materiais que possuem alto teor de carbono podem ser convertidos em carvão ativado e, por conseguinte, utilizados como adsorventes. ${ }^{17}$
Como exemplos, cita-se o carvão ativado produzido a partir de madeira e turfa (carvão fóssil), casca de coco e casca de arroz, ${ }^{5}$ bambu, casca de eucaliptos, ${ }^{7}$ acácia rubra, ${ }^{18}$ pedúnculo de algodão ${ }^{10}$ e o pedúnculo da uva. $^{19}$

Para a preparação do carvão ativado, os dois métodos mais utilizados são as ativações química ou física. A ativação física consiste na eliminação de impurezas, tais como os voláteis, por meio de pirólise/carbonização a fim de obter uma estrutura constituída basicamente por carbono. ${ }^{19-22}$

$\mathrm{Na}$ ativação química, que ocorre a temperaturas mais baixas com relação à ativação física, o processo ocorre na presença de agentes ativantes, tais como ácido fosfórico $\left(\mathrm{H}_{3} \mathrm{PO}_{4}\right)$, cloreto de zinco $\left(\mathrm{ZnCl}_{2}\right)$, hidróxido de potássio $(\mathrm{KOH})$, hidróxido de sódio $(\mathrm{NaOH})$, ácido clorídrico $(\mathrm{HCl})$, ácido sulfúrico $\left(\mathrm{H}_{2} \mathrm{SO}_{4}\right)$, entre outros. ${ }^{5,13,22-24}$ Estes agentes químicos utilizados são agentes de desidratação que influenciam na decomposição pirolítica e inibem a formação de alcatrão, aumentando o rendimento de carbono. $^{25}$

A ativação química apresenta algumas vantagens como menor temperatura de pirólise, maior rendimento e obtenção de material com maior área superficial específica. ${ }^{22,23,26}$

Nesse trabalho, são apresentados resultados de processos e testes aplicados na produção de um carvão ativado a partir de um resíduo agrícola, rico em carbono, a casca de noz. Foram utilizados dois métodos para preparação, a ativação física, por pirólise, e a ativação físico-química, com pirólise e tratamento químico. Sua eficiência como adsorvente para corantes foi testada utilizando o azul de metileno e os resultados foram comparados com o carvão ativado mineral. 


\section{Materiais e Método}

\subsection{Amostras}

As cascas de nozes foram obtidas de uma plantação no município de Criciúma, em Santa Catarina, na safra de 2013, oriundas de nogueiras-pecã (Carya illinoinensis). Foram secas em estufa a $110 \pm 5^{\circ} \mathrm{C}$ e trituradas até o tamanho de partícula entre 2 e $3 \mathrm{~mm}$.

\subsection{Preparação do carvão ativado}

As cascas de nozes in natura (CN-IN) foram trituradas em moinho planetário e colocadas em um cadinho de cordierita, o qual foi fechado com lã de rocha e uma placa de cordierita. O cadinho foi colocado em um forno tipo vertical (Jung 815) para o tratamento térmico à temperatura de $650 \pm 5$ ${ }^{\circ} \mathrm{C}$, a uma taxa de aquecimento de $10{ }^{\circ} \mathrm{C} \cdot \mathrm{min}^{-}$ ${ }^{1}$, durante $2 \mathrm{~h}$, para a pirólise. A temperatura do forno foi mantida no patamar máximo por $3 \mathrm{~h}$ e, na sequência, o forno foi resfriado com taxa de resfriamento de $10{ }^{\circ} \mathrm{C} \cdot \mathrm{min}^{-1}$ até a temperatura ambiente.

O carvão de casca de nozes foi moído durante $30 \mathrm{~min}$ em um moinho planetário, com esferas de alta alumina. As diferentes faixas de distribuição de tamanho de partícula foram separadas por peneiras com malha fixa, descartando-se a fração de tamanho de partícula maior que 0,149 mm, sendo este denominado de carvão ativado de cascas de nozes sem tratamento químico (CACN-ST).

Uma amostra desse material foi imersa em uma solução de cloreto de zinco - $\mathrm{ZnCl}_{2}$ (Merck), na concentração de $10 \% \mathrm{~m} / \mathrm{v}$ e na proporção de 1:5 (carvão:solução, massa:volume). A amostra foi imersa em solução e o sistema foi coberto com um filme plástico, permanecendo em repouso por 24 h. Decorrido este tempo, a amostra foi lavada com água destilada e seca em estufa a $110 \pm$ $5^{\circ} \mathrm{C}$.

O material lavado foi colocado em um cadinho de cordierita, fechado com lã de rocha e uma placa de cordierita, e pirolisado novamente por tratamento térmico, em forno tipo vertical (Jung 815), a $600 \pm 5{ }^{\circ} \mathrm{C}$, a uma taxa de $10{ }^{\circ} \mathrm{C} \cdot \mathrm{min}^{-1}$, sendo mantida nessa temperatura durante $2 \mathrm{~h}$. O carvão ativado foi lavado com uma solução de ácido clorídrico - $\mathrm{HCl}$ (Merck), a $2 \mathrm{~mol} \cdot \mathrm{L}^{-1}$, para remoção e desobstrução dos poros. Ao final do processo, a amostra foi seca em estufa a $110 \pm 5^{\circ} \mathrm{C}$ por $24 \mathrm{~h}$, e denominada de carvão ativado de cascas de nozes com tratamento químico (CACN-CT).

Para fins de comparação de resultados, utilizou-se carvão ativado (Synth), de tamanho de partícula inferior a $250 \mathrm{~mm}$, denominado de CA-STD.

\subsection{Caracterização do carvão ativado}

Para caracterização do carvão ativado produzido a partir da casca de noz, utilizou-se as técnicas de análise elementar de carbono, teor de cinzas, análise termogravimétrica (TGA), espectroscopia de infravermelho por transformada de Fourrier (FTIR), microscopia eletrônica de varredura (MEV) e área superficial (BET).

A análise elementar de carbono foi realizada em um analisador elementar por combustão CHNOS (LECO, CHN628), a partir das amostras maceradas.

O teor de cinzas do precursor (CN-IN) e dos materiais carbonáceos (CACN-ST, CACNCT e CA-STD) foi determinado pesando-se $1 \mathrm{~g}$ do material em um cadinho de porcelana previamente tarado e aquecendo-os em atmosfera oxidativa até $600 \pm 5{ }^{\circ} \mathrm{C}$ em mufla (Jung 815) por $3 \mathrm{~h}$. $\mathrm{O}$ experimento foi realizado em triplicata, sendo apresentados os resultados médios.

Para analisar o comportamento térmico dos carvões, as amostras de CN-IN, CACN-ST, CACN-CT e CA-STD foram submetidas à aquecimento em um analisador Shimadzu (TG-50), com taxa de aquecimento de 10 
${ }^{\circ} \mathrm{C} \cdot \mathrm{min}^{-1}$, sob fluxo de $10 \mathrm{~mL} \cdot \mathrm{min}^{-1}$ de $\mathrm{N}_{2}$, no intervalo de 25 a $1000{ }^{\circ} \mathrm{C}$.

$O$ ensaio de FTIR foi realizado para os materiais CN-IN, CACN-ST, CACN-CT e CASTD, na região do infravermelho, em pastilhas de $\mathrm{KBr}$, em um equipamento Shimadzu (IR Prestige-21), na faixa de 400 a $4000 \mathrm{~cm}^{-1}$, em intervalos de $2 \mathrm{~cm}^{-1}$.

A morfologia dos materiais foi avaliada por microscopia eletrônica de varredura (MEV), em um equipamento Shimadzu (ZeissMA10), com uma tensão entre 25 e 30 kV. A amostra foi colocada em um suporte de alumínio e uma fina camada de ouro foi aplicada em um evaporador Quorum Technologies-Q150R ES.

Para a determinação da distribuição do volume dos poros e da área superficial dos materiais carbonáceos, isotermas de adsorção e dessorção de $\mathrm{N}_{2}$ foram realizadas a 77,4 K, em um Autosorb-1 (Quantachrome). A distribuição do tamanho dos poros foi calculada pelo método $\mathrm{BJH}$, a partir da isoterma de adsorção. A área superficial foi calculada utilizando a equação BET na região de baixa pressão (p/po > 0,200).

A cinética de adsorção foi investigada em temperatura ambiente $\left(25 \pm 1^{\circ} \mathrm{C}\right)$ e $\mathrm{pH} 9,0 \pm$ 0,1 . Amostras de $100,00 \pm 0,01 \mathrm{mg}$ de carvão foram adicionadas a recipientes contendo $100 \pm 1 \mathrm{~mL}$ de solução de $25,0 \mathrm{mg} \cdot \mathrm{L}^{-1}$ do corante azul de metileno (AM). A amostra ficou em agitação constante e alíquotas do sobrenadante foram retiradas em intervalos predeterminados, e a concentração do adsorvente foi determinada a partir de análises de espectros de absorção de UV-Vis obtidos em um sistema BEL Photonics, LGS 53.

As isotermas de adsorção foram obtidas pela imersão de uma amostra de 0,1 g do carvão ativado em $100 \mathrm{~mL}$ de soluções de azul de metileno de concentrações entre 10 e $500 \mathrm{mg} \cdot \mathrm{L}^{-1}$. As misturas foram mantidas em agitação por $24 \mathrm{~h}$, à temperatura de $20 \pm 1$ ${ }^{\circ} \mathrm{C}$. A solução foi filtrada e a concentração foi determinada a partir de análises por espectrofotometria de absorção UV-Vis (BEL Photonics, LGS 53), no comprimento de onda de $645 \mathrm{~nm}$.

A massa adsorvida de material por unidade de massa do adsorvente $\left(q_{e q}\right)$ foi determinada aplicando a Equação (1), na qual $\mathrm{C}_{\mathrm{o}}$ e $\mathrm{C}_{\mathrm{eq}}$ representam as concentrações iniciais e no equilíbrio (em $\mathrm{mg} \cdot \mathrm{L}^{-1}$ ); $\mathrm{V}$ o volume da solução do corante (em L), e $m$ a massa do material adsorvente (em g). As isotermas foram ajustadas aos modelos de isotermas de Langmuir, apresentado na Equação (2), sendo $q_{e}$ a quantidade adsorvida por massa de adsorvente (em $\left.\mathrm{mg} \cdot \mathrm{g}^{-1}\right), \mathrm{C}_{\mathrm{e}}$ a concentração no equilíbrio (em $\mathrm{mg} \cdot \mathrm{L}^{-1}$ ) e $\mathrm{Q}_{\mathrm{o}} \mathrm{e}$ $b$ as constantes de Langmuir que relacionamse com a capacidade de adsorção e com a taxa de adsorção, respectivamente.

$$
\begin{aligned}
& q_{e q}=\frac{\left(C_{o}-C_{e q}\right) \cdot V}{m} \\
& \frac{1}{q_{e}}=\frac{1}{Q_{o} \cdot b \cdot C_{e}}+\frac{1}{Q_{o}}
\end{aligned}
$$

\section{Resultados e Discussões}

\subsection{Caracterização física e química}

As análises químicas revelaram que as amostras apresentaram baixo teor de cinzas, com valores de 6,7 e 7,2 \% para as amostras sem e com tratamento químico, respectivamente. Comparando estes valores com os obtidos com o carvão ativado de fonte mineral, o mesmo apresenta-se satisfatório, uma vez que o teor de cinzas para o material mineral padrão nesse trabalho é da ordem de $18 \%$.

Além disso, outro aspecto que favorece o uso de casca de nozes para produção de carvão ativado ao invés do carvão mineral é o efeito dielétrico. A presença de material mineral produz efeito dielétrico sobre o processo de adsorção e, devido ao caráter hidrofílico do material, adsorve preferencialmente a água, permanecendo a molécula do corante em água. ${ }^{17}$ Assim, 
quanto menor o teor de inorgânicos, maior a capacidade adsortiva.

Comparando o resultado obtido para o carvão ativado produzido e o seu precursor, a casca de noz, percebe-se um aumento esperado de teor de cinzas, uma vez que a casca de nozes in natura apresenta apenas 1,9 \% de material não volátil (inorgânico). 0 tratamento químico, associado à pirólise, não elevou consideravelmente o teor de cinzas, mostrando que o primeiro processo de carbonização foi eficiente na volatilização dos componentes orgânicos da casca de noz.
A análise de carbono elementar apresenta como resultado um teor de $79,6 \%$ para o CACN-ST e 78,4 \% para o CACN-CT. Uma pequena perda de massa em termos de teor de carvão é perceptível e deve-se ao segundo processo de carbonização da amostra. Ao ser novamente carbonizada, átomos de carbono sofrem oxidação, diminuindo o seu teor na amostra e, por consequência, aumentando o teor de carvão.

A Figura 1 apresenta os termogramas obtidos na análise termogravimétrica para as amostras CACN-ST, CACN-CT e CA-STD.

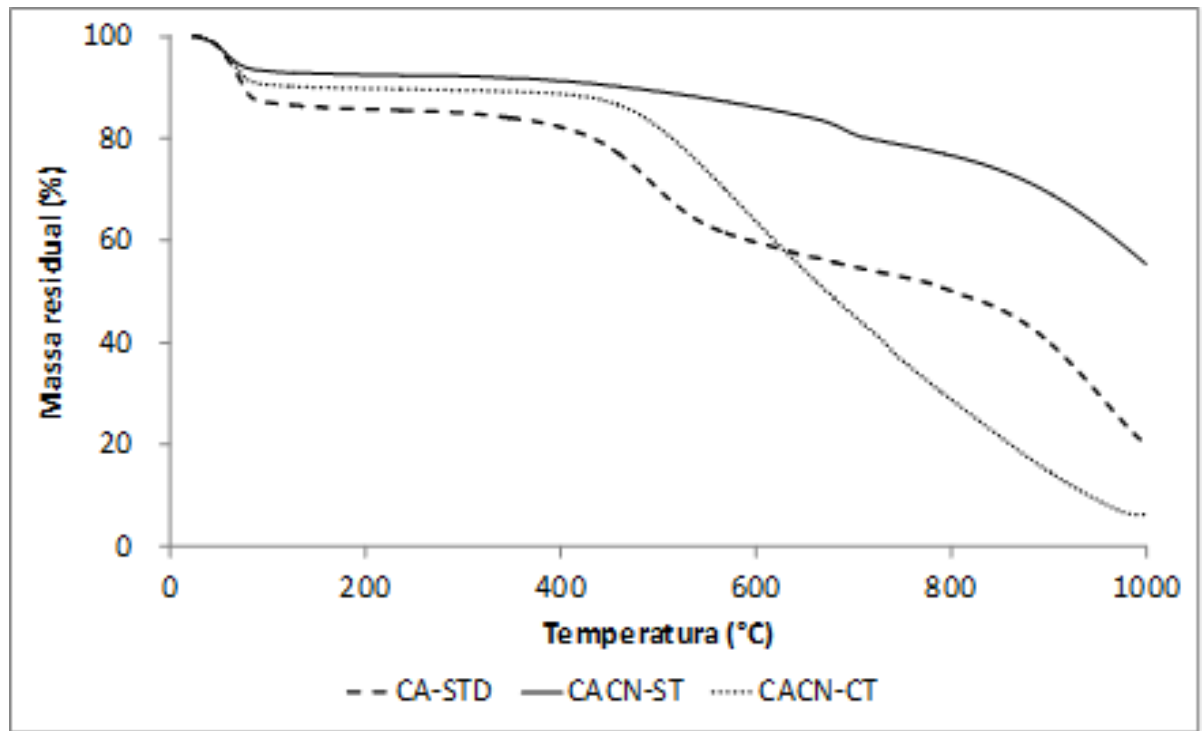

Figura 1. Termogramas de termogravimetria para as amostras do CACN-ST, CACN-CT e CASTD, por aquecimento a uma taxa de $10^{\circ} \mathrm{C} \cdot \mathrm{min}^{-1}$, sob fluxo de $10 \mathrm{~mL} \cdot \mathrm{min}^{-1}$ de $\mathrm{N}_{2}$, entre 25 e $1000{ }^{\circ} \mathrm{C}$

Nas amostras submetidas ao tratamento térmico, o primeiro momento envolvendo perda de massa ocorre próximo a $100{ }^{\circ} \mathrm{C}$. Este evento está associado com a perda de umidade existente nas amostras. $O$ segundo evento ocorre em temperaturas próximas de $450{ }^{\circ} \mathrm{C}$ e está relacionado com a liberação de compostos voláteis, constituídos por oxigênio e hidrogênio, como os compostos carboxilados remanescentes do processo da pirólise. ${ }^{24} \mathrm{~A}$ partir desta temperatura, a perda de massa do CACN-CT ocorre com taxa de perda de massa praticamente constante, resultando em um resíduo de, aproximadamente $7 \%$, como indicado na análise elementar. A amostra de CACN-ST apresentou um segundo gradiente de perda de massa próximo a $900{ }^{\circ} \mathrm{C}$ devido a uma provável aromatização dos átomos de carbono. $^{27}$

Comparado ao termograma da CA-STD, as amostras apresentaram menor estabilidade térmica, como esperado, uma vez que o material de origem mineral já passou por um processo de carbonização durante milhares de anos, em condições específicas de temperatura e pressão. No entanto, as 
amostras de carvão ativado a partir de cascas de nozes mostraram-se estáveis para aplicação como materiais adsorventes em efluentes industriais.
A Figura 2 apresenta o espectro de infravermelho para as amostras de $\mathrm{CN}-\mathrm{IN}$, CACN-ST, CACN-CT e CA-STD.

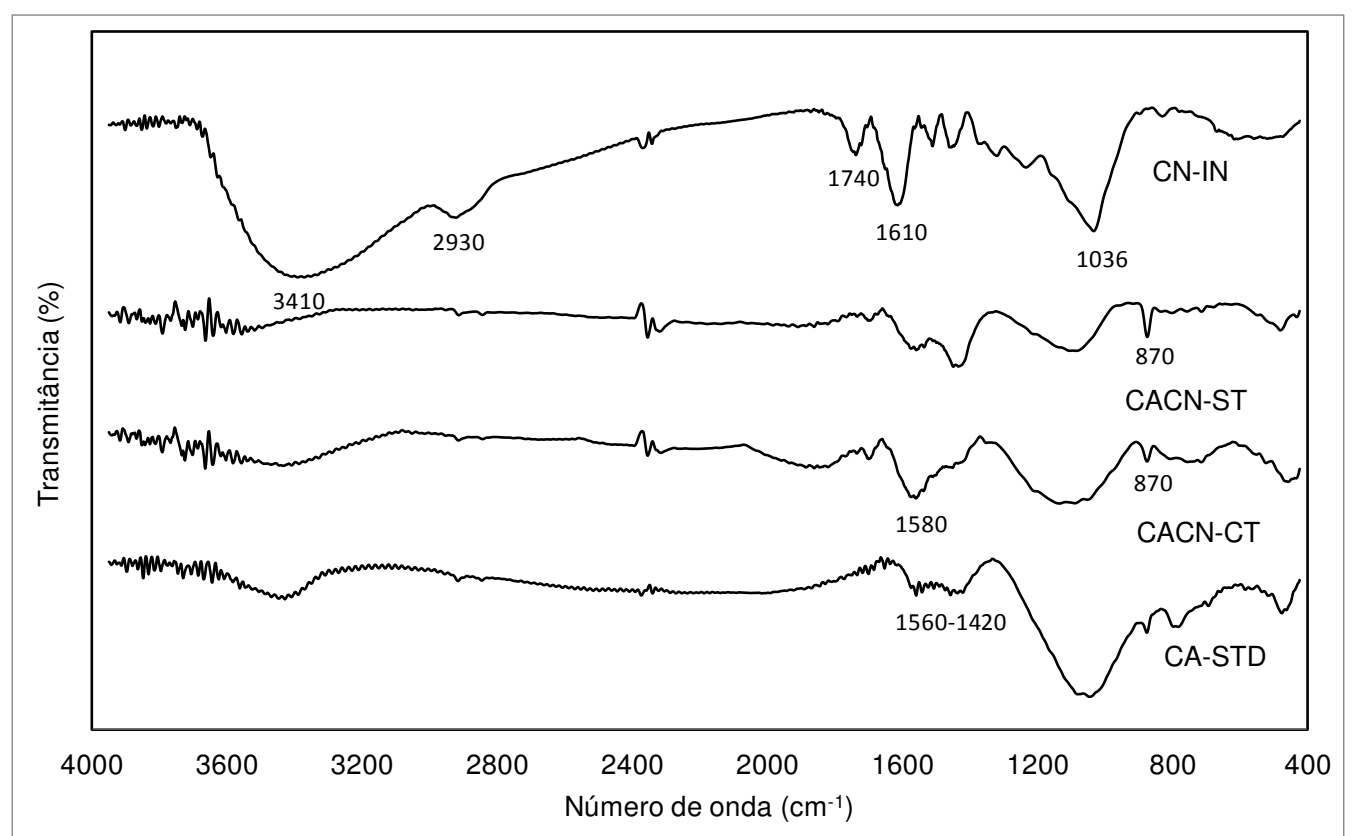

Figura 2. Espectros de infravermelhos obtidos por FTIR para a CN-IN, e os carvões CACN-ST, CACN-CT e CA-STD, em pastilha de $\mathrm{KBr}$

No espectro para a CN-IN é possível observar bandas características de materiais orgânicos oriundos de biomassa vegetal. A banda em torno de $3410 \mathrm{~cm}^{-1}$ é atribuída ao estiramento do grupo - $\mathrm{OH}$. As vibrações em torno de $2930 \mathrm{~cm}^{-1}$ representam ligações $\mathrm{C}-\mathrm{H}$ de hidrocarbonetos. ${ }^{5}$ As bandas em $1740 \mathrm{~cm}$ 1 e $1610 \mathrm{~cm}^{-1}$ evidenciam a presença de grupos carbonila $\mathrm{C}=\mathrm{O}$, existentes em ácidos carboxílicos, cetonas e na própria celulose. A absorção em $1036 \mathrm{~cm}^{-1}$ representa ligações C-O de álcoois.

Nos espectros relativos aos carvões ativados é possível perceber ausência ou considerável diminuição de bandas de grupos funcionais existentes no precursor. A banda atribuída à ligação $-\mathrm{OH}$ em torno de $3400 \mathrm{~cm}$ ${ }^{1}$ é um caso. ${ }^{28}$ Outro caso é a banda em torno de $2930 \mathrm{~cm}^{-1}$ que também, praticamente, deixa de existir. Com isso, fica clara a eficiência do processo de pirólise no material de base orgânica. ${ }^{5}$
As vibrações em torno de 1600 e $1400 \mathrm{~cm}$ ${ }^{1}$ existentes nas amostras de CNCA-ST, CACNCT e CA-STD são referentes ao estiramento de ligações $\mathrm{C}-\mathrm{C}$ e $\mathrm{C}=\mathrm{C}$ de aromáticos. $\mathrm{O}$ pico em torno de $870 \mathrm{~cm}^{-1}$ representa ligações de C-N. ${ }^{7}$

É possível perceber nos espectros uma inversão da magnitude entre as bandas próximas a $1580 \mathrm{~cm}^{-1}$ para as amostras de CACN-ST e CACN-CT. Estas bandas estão relacionadas às ligações $\mathrm{C}-\mathrm{C}_{\text {aromático. }}$. Portanto, sua inversão deve-se ao aumento de grau de aromaticidade de carbonos na amostra.

A Figura 3(a) apresenta a imagem capturada por microscopia eletrônica de varredura para o precursor, enquanto a Figura 3(b) mostra o carvão ativado sem tratamento químico e a Figura 3(c) ilustra o carvão ativado com tratamento químico.

O material precursor apresenta uma estrutura microscópica compacta, com sulcos celulósicos fibrosos. O processo de pirólise do 
material e sua ativação produzem modificações nessa estrutura e proporcionam o surgimento de poros na forma de pequenas cavidades devido à evaporação dos componentes voláteis que preenchiam os sulcos celulósicos no precursor. A estrutura do carvão adquirida é semelhante à estrutura obtida pela carbonização de outros materiais a base de celulose. ${ }^{4}$ Os pontos claros presentes na micrografia da Figura 3(b) evidenciam a presença de núcleos mais densos, provavelmente zinco residual do processo de tratamento químico.

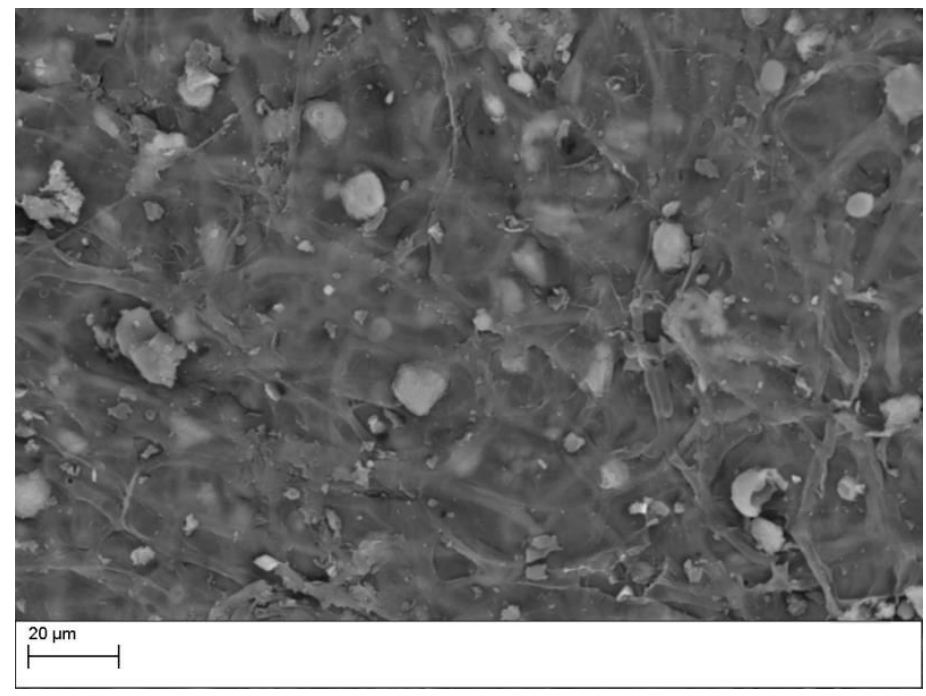

(a)

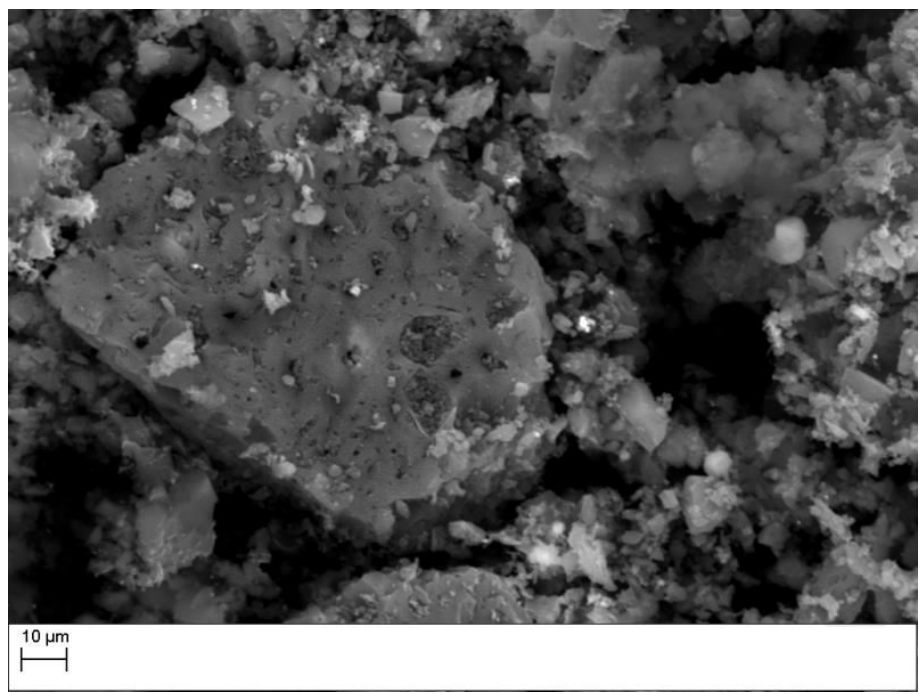

(b) 
de Costa, P. D. et al.

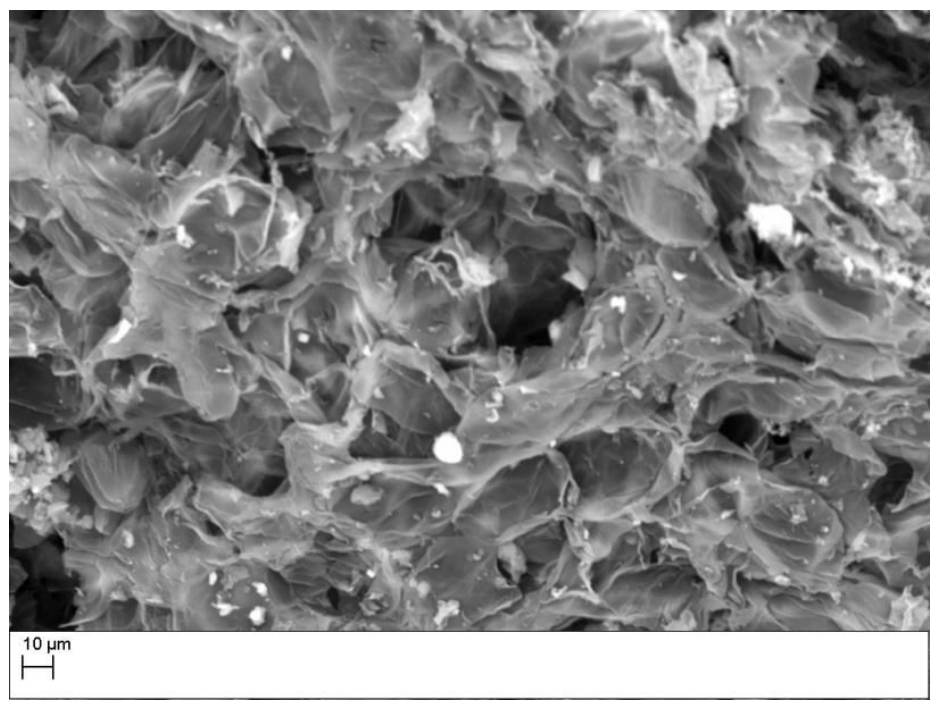

(c)

Figura 3. Micrografia para o (a) precursor, para o (b) CACN-ST e para o (c) CACN-CT, obtida em um microscópio eletrônico de varredura (Shimadzu, Zeiss, MA10)

A Figura 4 mostra as isotermas de adsorção/dessorção de $\mathrm{N}_{2}$ para os carvões produzidos a partir das cascas de nozes e também para o carvão de origem mineral.

As isotermas indicam que o carvão produzido a partir da pirólise das cascas de nozes apresenta a área superficial de 408 $\mathrm{m}^{2} \cdot \mathrm{g}^{-1}$. Após o tratamento químico com $\mathrm{ZnCl}_{2}$, a área superficial do carvão ativado passa a ser de $427 \mathrm{~m}^{2} / \mathrm{g}$, um acréscimo de, aproximadamente, $5 \%$. A área superficial do carvão produzido a partir das cascas de nozes é próxima a $80 \%$ da área superficial do carvão de origem mineral, um resultado considerado muito satisfatório.

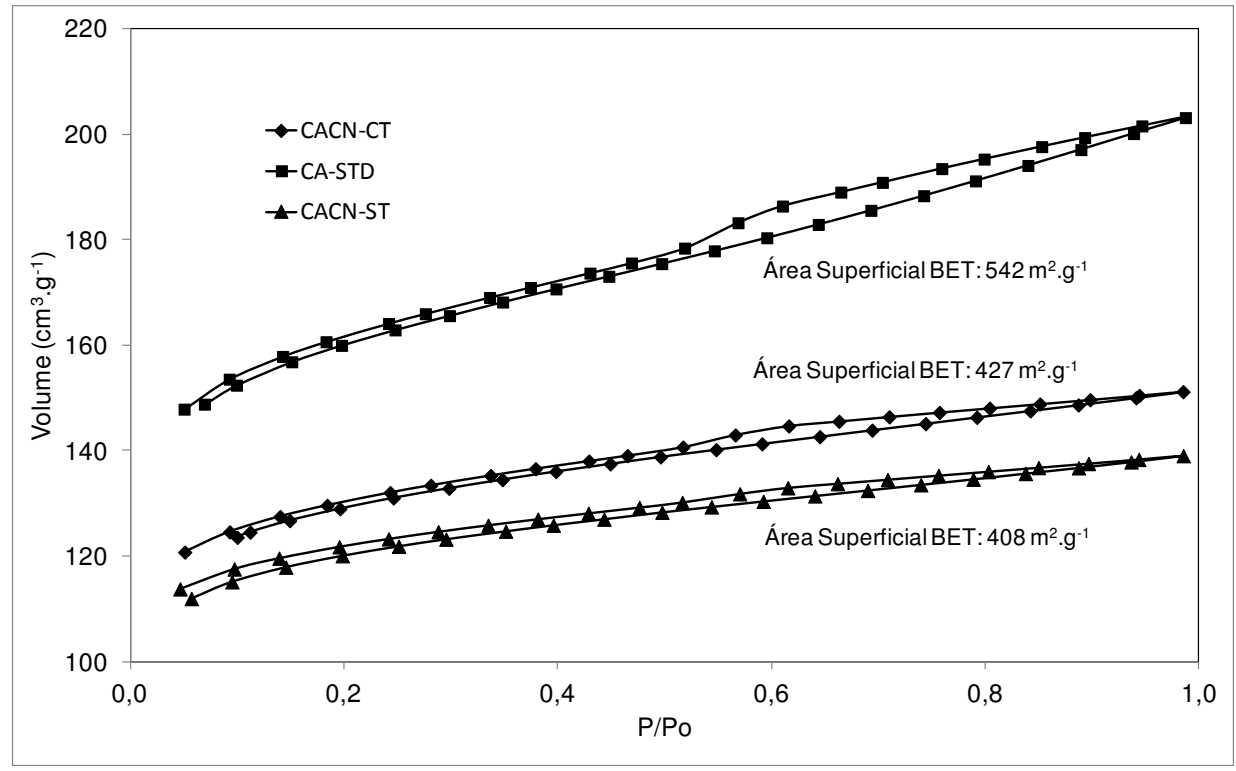

Figura 4. Isoterma de adsorção/dessorção de $\mathrm{N}_{2}$ para CACN-ST, CACN-CT e CACN-STD, obtida em um analisador BET, a 77,4 $\mathrm{K}$ 


\subsection{Cinética de adsorção}

A Figura 5 apresenta os resultados obtidos para os ensaios de cinética de adsorção empregando a técnica de absorção de UVVis. Percebe-se que a taxa de adsorção é elevada nos primeiros minutos e crescente na seguinte ordem das amostras: CACN-ST, CACN-CT e CA-STD.

A diminuição da taxa de adsorção com o tempo é um comportamento normal, considerando que, para a adsorção, o número de sítios ativos disponíveis nos tempos iniciais para a impregnação química do corante é maior, bem como a sua quantidade na solução. Com o passar do tempo, a quantidade de sítios ativos disponíveis para a adsorção das moléculas de corante diminuem. ${ }^{17}$ É possível observar que a condição de equilíbrio é atingida em tempo menor para o CA-STD, enquanto que, para os demais adsorventes, o equilíbrio é atingido após a primeira hora. Porém, para os três casos, a remoção é superior a $80 \%$ no período de tempo de, aproximadamente, 250 $\min$.

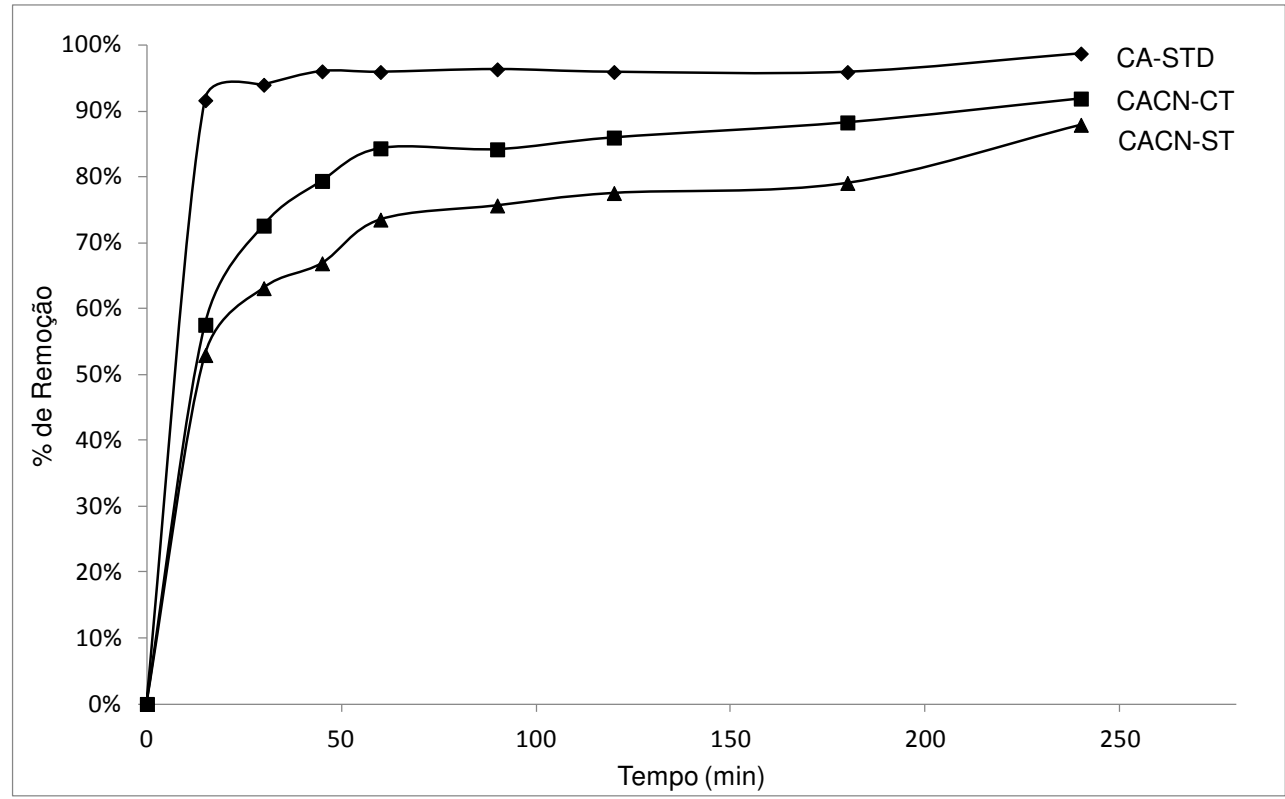

Figura 5. Diagramas de remoção por adsorção de AM pelo carvão ativado obtido a partir das cascas de nozes (100 mg.100 mL $\mathrm{L}^{-1}$ de solução, $25 \mathrm{mg} \cdot \mathrm{L}^{-1}$ )

\subsection{Isoterma de adsorção}

As isotermas de adsorção representam o resultado da interação entre o adsorvente e o adsorbato até o ponto de equilíbrio das fases. A Figura 6 ilustra os valores de $q_{e}$ para as concentrações de AM estudadas e para os dois carvões sintetizados comparados com o carvão de origem mineral. 


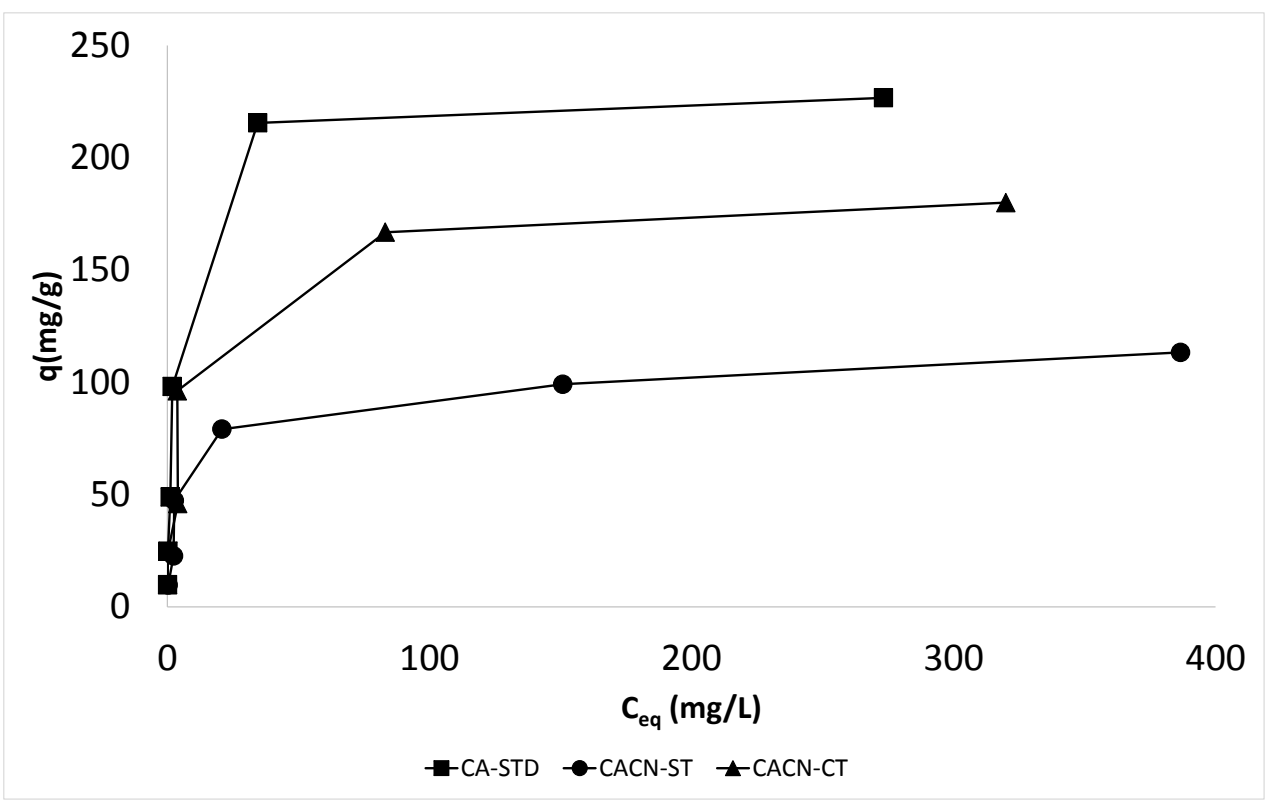

Figura 6. Isotermas de adsorção de AM pelas amostras de CACNs sem e com tratamento químico com $\mathrm{ZnCl}_{2}$, comparados ao CA-STD

Aplicando o modelo de isoterma de Langmuir, foram obtidos os valores de $Q_{0}$, sendo iguais a 68,104 e 112 para o CACN-ST, CACN-CT e CA-STD respectivamente, com $\mathrm{R}^{2}$ superiores a 0,95. De acordo com os valores obtidos, o CACN-CT se apresentou como o adsorvente mais eficaz em relação ao CACNST para remoção de AM de soluções aquosas.

Uma característica importante da isoterma de Langmuir é expressa em termos da constante adimensional denominada por $R_{L}$, determinada conforme a Equação (3), onde $b$ é a constante de Langmuir e Co é a concentração inicial do corante.

$$
R_{L}=\frac{1}{1+b \cdot C_{o}}
$$

Essa equação indica o tipo de isoterma. Para os testes realizados, os valores calculados de $R_{L}$ apresentaram-se entre $0<R_{L}$ $<1$, o que indica que a isoterma de adsorção é favorecida e constata que o modelo de
Langmuir se ajusta satisfatoriamente ao fenômeno de adsorção de AM por CACN nas condições estudadas. ${ }^{29}$

A Tabela 1 apresenta o comparativo entre alguns parâmetros obtidos nesse estudo com outros carvões ativados produzidos de maneira similar, a partir de resíduos carbonáceos.

Os resultados evidenciam, mais uma vez, que o CACN produzido é compatível para remoção de $A M$ e seus parâmetros são similares aos demais carvões ativados produzidos a partir de resíduos carbonáceos. No entanto, é possível perceber que, para tratamentos sem pirólise, somente com o uso de $\mathrm{H}_{2} \mathrm{SO}_{4}$, os resultados são melhores. ${ }^{30}$ Isso porque a não carbonização da amostra aumenta o teor de carbono no carvão ativado e, por consequência, sua área superficial. Porém, quando comparado à capacidade adsortiva $\left(\mathrm{mg} \cdot \mathrm{m}^{-2}\right)$, percebe-se que os resultados desse trabalho são tão satisfatórios quanto aos obtidos com o uso do carvão ativado comercial de origem mineral. 
Tabela 1. Comparativo entre o teor de cinza e de carbono elementar nas amostras de carvão ativado preparados a partir de resíduos de biomassa com ou sem ativação química

\begin{tabular}{|c|c|c|c|c|c|c|c|}
\hline Fonte primária & $\begin{array}{l}\text { Tratamento } \\
\text { químico }\end{array}$ & $\begin{array}{c}\text { Teor de } \\
\text { cinza (\%) }\end{array}$ & $\mathrm{C}(\%)$ & $\begin{array}{c}\text { Área } \\
\text { superficial } \\
\left(\mathrm{m}^{2} \cdot \mathrm{g}^{-1}\right)\end{array}$ & $\underset{\left(m g \cdot g^{-1}\right)}{q_{e q}}$ & $\begin{array}{c}\text { Capacidade } \\
\text { adsortiva } \\
\left(\mathrm{mg} \cdot \mathrm{m}^{-2}\right)\end{array}$ & Fonte \\
\hline CA-STD mineral & - & 18,6 & 65,8 & 542 & 112 & 0,207 & Synth \\
\hline \multirow{2}{*}{ Casca de nozes } & Sem & 6,7 & 79,6 & 408 & 68 & 0,167 & \multirow{2}{*}{$\begin{array}{l}\text { Este } \\
\text { estudo }\end{array}$} \\
\hline & $\mathrm{ZnCl}_{2}$ & 7,2 & 78,4 & 427 & 104 & 0,244 & \\
\hline $\begin{array}{c}\text { Grãos } \\
\text { defeituosos de } \\
\text { café }\end{array}$ & $\mathrm{ZnCl}_{2}$ & 20,0 & 67,0 & 516 & 102 & 0,198 & 17 \\
\hline $\begin{array}{c}\text { Resíduos de } \\
\text { beneficiamento } \\
\text { de café }\end{array}$ & $\mathrm{ZnCl}_{2}$ & 1,4 & 33,8 & 522 & 188,7 & 0,361 & 28 \\
\hline Casca de arroz & $\mathrm{ZnCl}_{2}$ & - & - & 180 & 10,62 & 0,059 & 30 \\
\hline Casca de coco & $\mathrm{ZnCl}_{2}$ & - & 77,1 & 1884 & 14,36 & 0,008 & 31 \\
\hline $\begin{array}{l}\text { Cascas de } \\
\text { amêndoas }\end{array}$ & \multirow{4}{*}{$\mathrm{H}_{2} \mathrm{SO}_{4}$} & 2,8 & 87,9 & 998 & 261 & 0,262 & \multirow{4}{*}{32} \\
\hline Casca de nozes & & 4,9 & 85,9 & 743 & 222 & 0,299 & \\
\hline Sementes de uva & & 13,1 & 84,7 & 497 & 183 & 0,368 & \\
\hline $\begin{array}{l}\text { Cascas de } \\
\text { damasco }\end{array}$ & & 2,0 & 89,5 & 1190 & 316 & 0,266 & \\
\hline
\end{tabular}

Os estudos comprovam a possibilidade de uso das cascas de nozes para produção de carvão ativado com elevada área superficial (acima de $\left.400 \mathrm{~m}^{2} \cdot \mathrm{g}^{-1}\right)$, baixo teor de cinza $(6,7$ a $7,2 \%$ ) e elevado teor de carbono (acima de $78 \%)$.

Os ensaios de FTIR indicam a presença de grupos orgânicos essenciais para a interação com os sítios ativos de um corante catiônico, bem como as micrografias evidenciam a formação de uma estrutura tipo favo, que favorece o aumento da área superficial do carvão.

Os experimentos constataram que o $\mathrm{CACN}$ tem potencial para remoção de $A M$ de solução aquosa e que o tratamento com $\mathrm{ZnCl}_{2}$ aumenta a sua capacidade de adsorção de 68 para $104 \mathrm{mg} \cdot \mathrm{g}^{-1}$. Vale ainda comentar que o modelo de isoterma de Langmuir apresentou boa correlação e se ajusta com os resultados nas condições apresentadas.

\section{Agradecimentos}

Ao Instituto de Engenharia e Tecnologia da Universidade do Extremo Sul Catarinense (UNESC), ao Departamento de Pesquisa do Instituto Federal de Santa Catarina (IFSC) e à Universidade Estadual de Ponta Grossa (UEPG), pela disponibilização dos espaços físicos e equipamentos para ensaios.

\section{Referências Bibliográficas}

${ }^{1}$ O'Neill, C.; Hawkes, F. R.; Hawkes, D. L.; Lourennço, N. D.; Pinheiro, H. M.; Delée, W. Colour in textile effluents - sources, measurement, discharge consents and simulation: a review. Journal Chemical Technology and Biotechnology 1999, 74, 1009. [CrossRef] 
${ }^{2}$ Kunz, A.; Peralta-Zamora, P.; de Moraes, S. G.; Durán, N. Novas tendências no tratamento de efluentes têxteis. Química Nova 2002, 25, 78. [CrossRef]

${ }^{3}$ da Rocha, O. R. S.; do Nascimento, G. E.; Campos, N. F.; da Silva, V. L.; Duarte, M. M. M. B. Avaliação do processo adsortivo utilizando mesocarpo de coco verde para remoção do corante cinza reativo BF-2R. Química Nova 2012, 35, 1369. [CrossRef]

${ }^{4}$ Cherifi, H.; Fatih, B; Salah, H. Kinetic studies on the adsorption of methylene blue onto vegetal fiber activated carbons. Applied Surface Science 2013, 282, 52. [CrossRef]

${ }^{5}$ Pereira, E.; Oliveira, L. C. A.; Vallone, A.; Sapag, K.; Pereira, M. Preparação de carvão ativado em baixas temperaturas de carbonização a partir de rejeitos de café: utilização de $\mathrm{FeCl}_{3}$ como agente ativante. Química Nova 2008, 31, 1296. [CrossRef]

${ }^{6}$ Sud, D.; M., Mahajan, G.; Kaur, M. P. Agricultural waste material as potential adsorbent for sequestering heavy metal ions from aqueous solutions - A review. Bioresource Technology 2008, 99, 6017. [CrossRef] [PubMed]

${ }^{7}$ Pathania, D.; Sharma, S.; Singh, P. Removal of methylene blue by adsorption onto activated carbon developed from Ficus carica bast. Arabian Journal of Chemistry 2014, no prelo. [CrossRef]

${ }^{8}$ Rafatullah, M.; Sulaiman, O.; Hashim, R.; Ahmad, A. Adsorption of methylene blue on low-cost adsorbents: A review. Journal of Hazardous Materials 2010, 177, 70. [CrossRef] [PubMed]

${ }^{9}$ Bazrafshan, E.; Mostafapour, F. K.; Zazouli, M. A. Methylene blue (cationic dye) adsorption into Salvadora persica stems ash. African Journal of Biotechnology 2012, 11, 16661. [CrossRef]

${ }^{10}$ Deng, H.; Lu, J.; Li, G.; Zhang, G.; Wang, X. Adsorption of methylene blue on adsorbent materials produced from cotton stalk. Chemical Engineering Journal 2011, 172. 326. [CrossRef]

${ }^{11}$ Park, S. J.; Kim, K. D. J. Adsorption behaviors of $\mathrm{CO}_{2}$ and $\mathrm{NH}_{3}$ on chemically surface treated activated carbons. Journal Colloid Interface Science 1999, 212, 186. [CrossRef] [PubMed]
12 Pezoti Junior, O.; Cazetta, A. L.; Gomes, R. C.; Barizão, E. O. J.; Souza, I. P. A. F.; Martins, A. C.; Asefa, T.; Almeida, V. C. Synthesis of $\mathrm{ZnCl}$-activated carbon from macadamia nut endocarp (Macadamia integrifolia) by microwave-assisted pyrolysis: Optimization using RSM and methylene blue adsorption. Journal of Analytical and Applied Pyrolysis 2014, 105, 1660. [CrossRef]

${ }^{13}$ Guo, Y.; Rockstraw, D. A. Physicochemical properties of carbons prepared from pecan shell by phosphoric acid activation. Bioresource Technology 2007, 98, 1513. [CrossRef] [PubMed]

${ }^{14}$ Bueno, C. I. C.; Carvalho, W. A. Remoção de Chumbo (II) em sistemas descontínuos por carvões ativados com ácido fosfórico e com vapor. Química Nova 2007, 30, 1911. [CrossRef]

${ }^{15}$ Dursun, G.; Çiçek, H.; Dursun ARZU, Y. J. Adsorption of phenol from aqueous solution by using carbonised beet pulp. Journal of Hazardous Materials 2005, 125, 175. [CrossRef] [PubMed]

${ }_{16}$ Barbosa, C. S.; Santana, S. A. A.; Bezerra, C. W. B.; Silva, H. A. dos S. Remoção de compostos fenólicos de soluções aquosas utilizando carvão ativado preparado a partir do aguapé (Eichhornia crassipes): estudo cinético e de equilíbrio termodinâmico. Química Nova 2014, 37, 447. [CrossRef]

${ }^{17}$ Ramos, P. H.; Guerreiro, M. C. Resende, E. C. de; Golçalves, M. Produção e caracterização de carvão ativado produzido a partir do defeito preto, verde, ardido (PVA) do café. Química Nova 2009, 32, 1139. [CrossRef]

${ }^{18}$ Vargas, A. M. M.; Cazetta, A. L.; Kunita, M. H. Silva, T. L. Almeida, V. C. Adsorption of methylene blue on activated carbon produced from flamboyant pods (Delonix regia): study of adsorption isotherms and kinetic models. Chemical Engineering Journal 2011, 168, 722. [CrossRef]

${ }^{19}$ Ozdemir, I.; Sahin, M.; Orhan, R.; Erdem, M. Preparation and characterization of activated carbon from grape stalk by zinc chloride activation. Fuel Processing Technology 2014, 125, 200. [CrossRef]

${ }^{20}$ Foo, K. Y.; Hameed, B. H. Adsorption characteristics of industrial solid waste 
derived activated carbon prepared by microwave heating for methylene blue. Fuel Processing Technology 2012, 99, 103. [CrossRef]

${ }^{21}$ Salleh, M. A. M.; Mahmoud, D. K.; Karin, M. A. A.; Idris, A. Cationic and anionic dye adsorption by agricultural solid wastes: A comprehensive review. Desalination 2011, 280, 1. [CrossRef]

${ }^{22}$ Ahmadpour, A.; Do, D. D. The preparation of active carbons from coal by chemical and physical activation. Carbon 1996, 34, 471. [CrossRef]

${ }^{23}$ Gerçel, Ö.; Özcan, A.; Özcan, A. S.; Gerçel, H. F. Preparation of activated carbon from a renewable bio-plant of Euphorbia rigida by $\mathrm{H}_{2} \mathrm{SO}_{4}$ activation and its adsorption behavior in aqueous solutions. Applied Surface Science 2007, 253, 4843. [CrossRef]

${ }^{24}$ Zaini, M. A. A.; Zakaria, M.; Mohd.-Setapar, S. H.; Che-Yunus, M. A. J. Sludge-adsorbents from palm oil mill effluent for methylene blue removal. Journal of Environmental Chemical Engineering. 2013, 1, 1091. [CrossRef]

${ }^{25}$ Kandiyoti, R.; Lazaridis, J. I.; Dyrvold, B.; Weerasinghe, R. Pyrolysis of a $\mathrm{ZnCl}_{2-}$ impregnated coal in an inert atmosphere. Fuel 1984, 63, 1583. [CrossRef]

${ }^{26}$ Mohanty, K.; Das, D.; Biswas, M. N. Adsorption of phenol from aqueous solutions using activated carbons prepared from Tectona grandis sawdust by $\mathrm{ZnCl}_{2}$ activation. Chemical Engineering Journal 2005, 115, 121. [CrossRef]

${ }^{27}$ Rodriguez-Reinoso, F.; Molina-Sabio, M.; Gonzalez, M. T. The use of steam and $\mathrm{CO}_{2}$ as activating agents in the preparation of activated carbons. Carbon 1995, 33, 15. [CrossRef]

${ }^{28}$ Brum, S. S.; Bianchi, M. L.; da Silva, V. L.; Gonçalves, M.; Guerreiro, M. C.; de Oliveira, C. A. Preparação e caracterização de carvão ativado produzido a partir de resíduos do beneficiamento do café Química Nova, 2008, 31, 1048. [CrossRef]

${ }^{29}$ Zhang, Z.; Zhang, Z.; Fernández, Y.; Menéndez, J. A.; Niu, H.; Peng, J.; Zhang, L.; Guo, S. Adsorption isotherms and kinetics of methylene blue on a low-cost adsorbent recovered from a spent catalyst of vinyl acetate synthesis. Applied Surface Science 2010, 256, 2569. [CrossRef]

${ }^{30}$ Sharma, Y. C.; Uma; Upadhyay, S. N. Can. An economically viable removal of methylene blue by adsorption on activated carbon prepared from rice husk. The Canadian Journal of Chemical Engineering 2011, 89, 377 [CrossRef]

${ }^{31}$ Macedo, J. S.; Junior, N. B. C.; Almeida, L. E.; Vieira, E. F. S.; Cestari, A. R.; Gimenez, I. F.; Carreño, N. L. V.; Barreto, L. S. Kinetic and calorimetric study of the adsorption of dyes on mesoporous activated carbon prepared from coconut coir dust. Journal of Colloid and Interface Science 2006, 298, 515 [CrossRef] [PubMed]

${ }^{32}$ Savova, D.; Apak, E.; Ekinci, E.; Yardim, F.; Petrov, N.; Budinova, T.; Razvigorova, M.; Minkova, V. Biomass conversion to carbon adsorbents and gas. Biomass and Bioenergy 2001, 21, 133. [CrossRef] 